\title{
Spontaneous Reporting to Regulatory Authorities of Suspected Adverse Drug Reactions to COVID-19 Vaccines Over Time: The Effect of Publicity
}

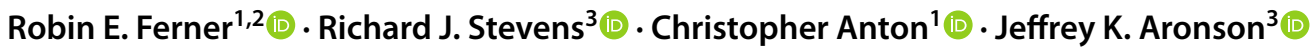

Accepted: 8 December 2021 / Published online: 22 January 2022

(c) The Author(s), under exclusive licence to Springer Nature Switzerland AG 2022

\begin{abstract}
Introduction The UK Medicines and Healthcare products Regulatory Agency (MHRA) has published frequent summaries of spontaneous reports of suspected adverse drug reactions (ADRs) (Yellow Cards) to vaccines against severe acute respiratory syndrome coronavirus 2 (SARS-CoV-2). The EudraVigilance database has provided similar data for the European Economic Area.

Objective Our objective was to characterize the evolution over time of spontaneous reports of suspected ADRs to coronavirus disease 2019 (COVID-19) vaccines and to observe the effect of a publicized reaction (cerebral venous and sinus thrombosis [CVST]) on reporting rates.

Methods We used publicly available data on reports of suspected ADRs and doses of vaccine administered, published by the MHRA, EudraVigilance, and the European Centre for Disease Prevention and Control to calculate reporting rates.

Results Approximately 4814 Yellow Card reports (23 fatal) per million doses of ChAdOx1 nCoV-19 (AstraZeneca) and 2890 (13 fatal) per million doses of tozinameran (Pfizer/BioNTech) have been lodged. Between 15 March and 31 October 2021, cumulative European reports of CVST rose from 0 to 443 (183 with thrombocytopenia, 72 fatal) with ChAdOx 1 nCoV-19 and from 2 to 315 (9 with thrombocytopenia, 28 fatal) with tozinameran. European cases of retinal vein occlusion and thrombosis rose from 0 to 168 with ChAdOx $1 \mathrm{nCoV}-19$ and from 1 to 220 with tozinameran; four of the ChAdOx 1 $\mathrm{nCoV}-19$ cases were associated with thrombocytopenia.

Conclusion Reports of fatal adverse reactions to coronavirus vaccines are very rare. Reports of CVST have been made in relation to both vaccines. Most were submitted after the reaction had been publicized. Thrombocytopenia occurred in a minority of cases. Reports linked both vaccines to cases of retinal vein thrombosis, just four cases with thrombocytopenia. This suggests two different mechanisms of thrombosis associated with the vaccines.
\end{abstract}

Robin E. Ferner

r.e.ferner@bham.ac.uk

1 West Midlands Centre for Adverse Drug Reactions, City Hospital, Birmingham B18 7QH, UK

2 Institute of Clinical Sciences, University of Birmingham, Edgbaston, Birmingham, UK

3 Centre for Evidence-Based Medicine, Nuffield Department of Primary Care Health Sciences, Medical Science Division, University of Oxford, Oxford, UK 


\section{Key Points}

Reporting rates for the AstraZeneca vaccine have remained higher than those for the Pfizer vaccine, although reports of suspected fatal reactions to either vaccine are very rare.

The reporting rate for cerebral venous and sinus thrombosis increased rapidly after this possible adverse reaction was publicized, but fewer than half the cases are associated with thrombocytopenia.

There may be two separate mechanisms of venous thrombosis, only one of which is related to thrombocytopenia.

\section{Introduction}

Two vaccines against severe acute respiratory syndrome coronavirus 2 (SARS-CoV-2) are widely used in the UK: tozinameran (Comirnaty ${ }^{\circledR}$, Pfizer/BioNTech) and ChAdOx1 nCoV-19 (Vaxzevria ${ }^{\circledR}$, COVID-19 AstraZeneca Vaccine, AstraZeneca). Both are highly effective in preventing severe coronavirus disease 2019 (COVID-19) [1, 2]. They are also, by the standards usually applied, remarkably free from serious adverse effects. Adverse events after administration of tozinameran were assessed, before temporary regulatory approval, in over 18,000 subjects who received two doses of the vaccine in a randomized placebo-controlled trial [1]. Two patients in the treatment arm and four in the placebo arm died; none of the deaths was suspected to have been caused by the intervention. A pooled analysis assessed harms in over 12,000 people who had received ChAdOx1 nCoV-19 and nearly 12,000 placebo-treated control subjects [2]. Serious adverse events were recorded in 79 participants in the active treatment group and 89 in the control group. One non-COVID-19 death occurred in the vaccine group and three in the control group; none was considered related to the vaccine.

In these randomized trials, fewer than 1 in 3000 people died, and no death was attributed to the vaccine. If there were no fatalities in 12,000 people randomized to receive ChAdOx $1 \mathrm{nCoV}-19$, then the upper limit of a $95 \%$ confidence interval for fatal adverse reactions is 1 in 4000 [3]. Similarly, an upper confidence bound for fatal adverse reactions to tozinameran is 1 in 6000 . Adverse reactions are classed as rare according to standard definitions if they occur in fewer than 1 in 1000 people, and very rare in fewer than
1 in 10,000 [4]. Such adverse events would not be detected in trials of conventional size.

The data on vaccines against COVID-19 are unusually accessible, because information on both spontaneous reports of adverse events that are suspected of being adverse drug reactions (ADRs) and the numbers of doses administered have been published frequently. Such data can provide information on reporting in relation to administration [5].

ChAdOx $1 \mathrm{nCoV}-19$, developed in Oxford and widely used in the UK, later attracted criticism in continental Europe. On 10 March 2021, the European Medicines Agency (EMA) issued a news bulletin in which they reported three thromboembolic events in Austria after administration of ChAdOx1 nCoV-19 [6]. On 11 May 2021, the UK national news media reported that Denmark and Norway had suspended use of the vaccine [7], as many other European countries did subsequently, although not the UK. A particular concern was cerebral venous and sinus thrombosis (CVST) as part of the syndrome of vaccine-induced thrombosis and thrombocytopenia (VITT) [8]. The syndrome resembles heparin-induced thrombosis and thrombocytopenia (HITT). Both conditions are associated with antibodies to platelet factor four [9]. VITT has been particularly associated with CVST [10], which is otherwise very rare, although it is recognized to occur in HITT [11]. The UK Medicines and Healthcare products Regulatory Agency (MHRA) stated in May 2021 that the "evidence to date does not suggest that the COVID-19 Vaccine AstraZeneca causes venous thromboembolism without a low platelet count" [12]. We therefore sought to establish reporting rates of CVST with and without thrombocytopenia.

Retinal vein thrombosis and retinal vein occlusion, potential thrombotic complications, are rarely reported in association with HITT [13]. It was therefore of interest to establish the frequency of reports of retinal vein occlusion or thrombosis (RVT) with and without thrombocytopenia as a suspected ADR to vaccines against SARS-CoV-2.

We hypothesised that the reporting rate might be affected by administration of a second dose, either because people sensitized by the first dose might experience worse reactions or because severe reactions with the first dose might have precluded a second dose.

The MHRA summaries list only numbers of reactions and of reports (which can include more than one reaction). They do not include associated demographic data; nor do they link reactions to specific reports. We therefore also examined data from the EMA EudraVigilance database for the two vaccines [14], restricted to reports designated as coming from the European Economic Area (EEA, i.e. the EU plus Norway, Iceland, and Liechtenstein). The population of the EU 
is approximately 447 million [15] and of Norway, Iceland, and Liechtenstein combined approximately 5.8 million [16].

\section{Methods}

We used data published by the MHRA, the EMA, and the European Centre for Disease Prevention and Control.

We calculated reporting rates for each vaccine by dividing the number of reports of suspected ADRs by the number of doses of vaccine administered.

We collected data on Yellow Card reports of suspected adverse reactions to ChAdOx $1 \mathrm{nCoV}-19$ and tozinameran from the MHRA's summary of coronavirus vaccines issued each week from 24 January 2021 (the first collection date for data in the MHRA summary) to 27 October 2021 [12]. The numbers of first and second doses of a vaccine were taken from the MHRA summary. The numbers of suspected reactions and reports were taken from the relevant Vaccine Analysis Prints for ChAdOx1 nCoV-19 and tozinameran $[17,18]$, referred to in the weekly update.

We looked specifically for reports of cerebral venous thrombosis, cerebral venous sinus thrombosis, superior sagittal sinus thrombosis, and transverse sinus thrombosis. We also looked at reports of retinal vein thrombosis and retinal vein occlusion.

We examined whether reporting rates changed following the wide publicity given to reports that ChAdOx 1 nCoV-19 was associated with clotting abnormalities by comparing the rates of total and fatal reports per million doses for the weeks up to 14 March 2021 and from 23 March 2021 to 27 October 2021 for all reports and reports of suspected cerebral venous and sinus thrombosis and of RVT. We used data from EudraVigilance to assess how often patients in whom thrombotic events had been reported also reportedly had thrombocytopenia.

\section{Results}

\subsection{UK Data}

The MHRA data run from 4 January 2021 for ChAdOx 1 nCoV-19 and from 9 December 2020 for tozinameran. Approximately 4814 Yellow Card reports (23 fatal) per million doses of ChAdOx $1 \mathrm{nCoV}-19$ (AstraZeneca) and 2890 (13 fatal) per million doses of tozinameran (Pfizer/BioNTech) were logged. The rate of new reports of suspected ADRs fluctuated between 2620 and 10283 total reports per million new doses for ChAdOx1 nCoV-19 and between 1339 and 9197 total reports per million doses for tozinameran over the summaries issued once a week (Fig. 1). The rates of reported reactions did not appear to change markedly as the proportion of second doses increased (Fig. 1).

Reports of fatal events that were suspected to be ADRs (fatal reports) in the MHRA data varied between 7 and 56 reports per million doses for ChAdOx $1 \mathrm{nCoV}-19$ and

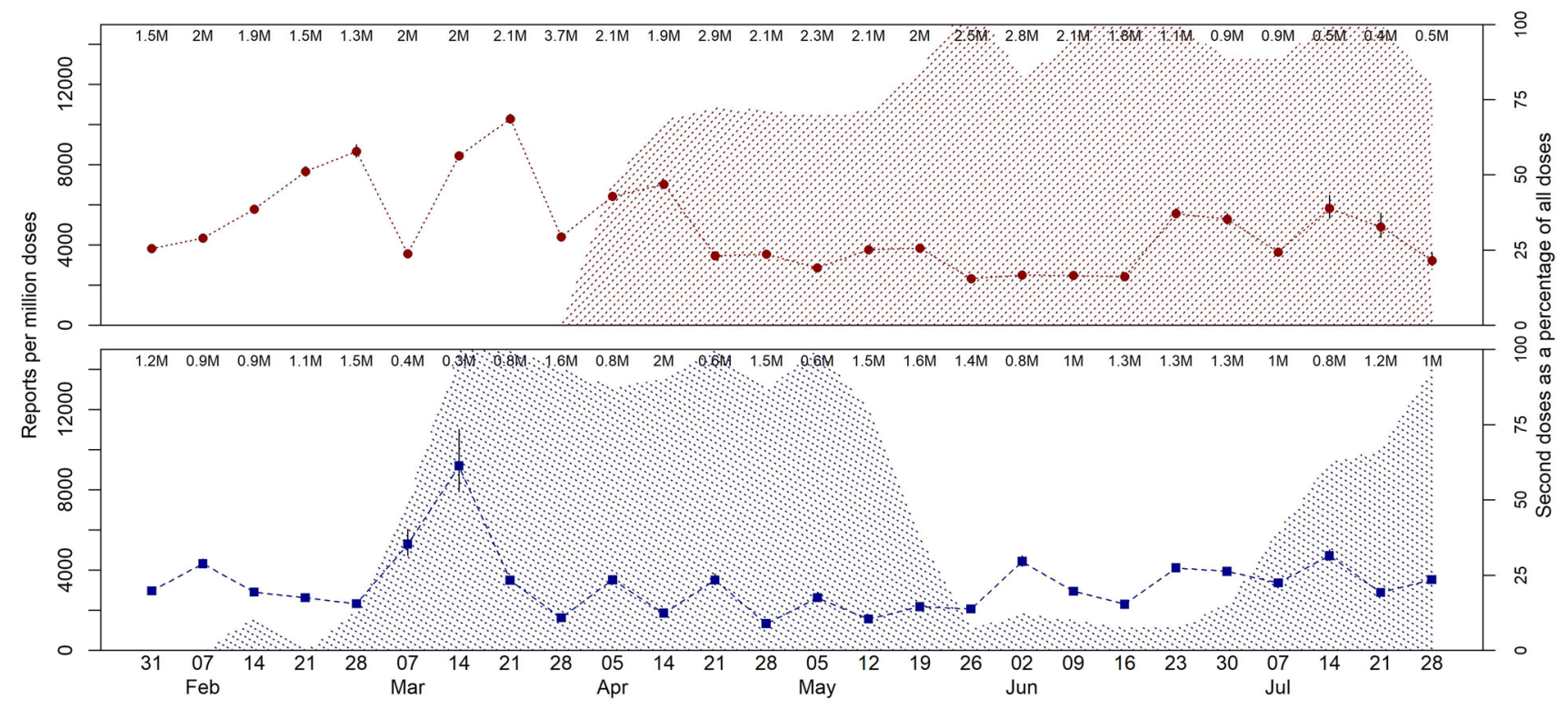

Fig. 1 Yellow Card reports of all suspected adverse drug reactions received in each time interval per million doses administered (lefthand axis). The number of doses administered each week is shown above the graphs. Error bars denote the imprecision due to rounding errors in the denominator, which is reported to the nearest 100,000 doses. The hatched shading shows second dose as a percentage of all doses (right-hand axis). $\mathrm{M}$ million, circles indicate $\mathrm{ChAdOx} 1 \mathrm{nCoV}$ 19, squares indicate tozinameran 
Fig. 2 Yellow Card reports of fatal suspected adverse drug reactions received in each time interval, per million doses administered in that time interval. Error bars denote the imprecision due to rounding errors in the denominator, which is reported to the nearest 100,000 doses. Circles indicate ChAdOx1 nCoV-19, squares indicate tozinameran

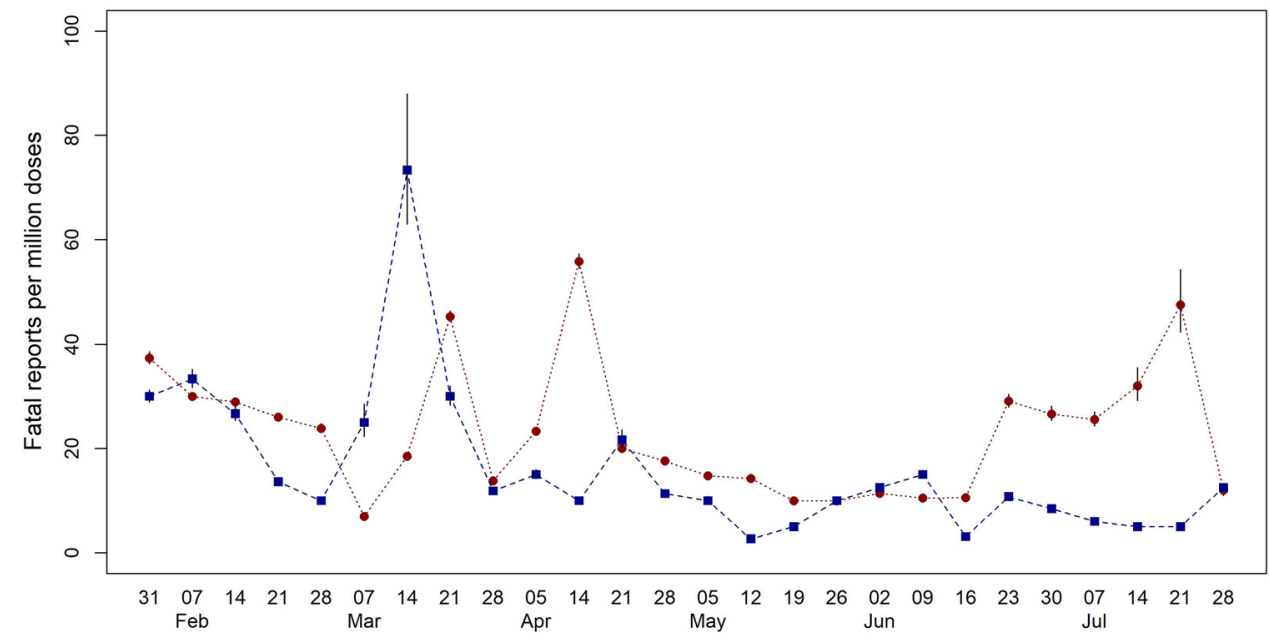

\begin{tabular}{|c|c|c|}
\hline Vaccine & ChAdOx1 nCoV-19 & Tozinameran \\
\hline Initial date of regulatory authorization & 4 January 2021 & 9 December 2020 \\
\hline \multicolumn{3}{|l|}{ Number of doses administered (millions) } \\
\hline First & 24.9 & 23.5 \\
\hline Second & 24.1 & 20.3 \\
\hline Total doses given & 49.0 & 43.8 \\
\hline \multicolumn{3}{|l|}{ Number of reports } \\
\hline Total reports & 235,875 & 126,603 \\
\hline Total reports/million doses & 4814 & 2890 \\
\hline Fatal & 1112 & 577 \\
\hline Fatal/million doses & 23 & 13 \\
\hline Percentage fatal & $0.47 \%$ & $0.46 \%$ \\
\hline
\end{tabular}

Reports can list several reactions, each of which is counted separately in the database

MHRA UK Medicines and Healthcare products Regulatory Agency
Table 1. Reports of suspected adverse reactions to $\mathrm{ChAdOx}$ nCoV-19 (AstraZeneca) and tozinameran (Pfizer/BioNTech) from the MHRA Yellow Card database (from licensing to 27 October 2021)
Table 2 Suspected adverse reactions to ChAdOx1 nCoV-19 (AstraZeneca) and tozinameran (Pfizer/BioNTech) from the MHRA Yellow Card database (from licensing to 27 October 2021)

\begin{tabular}{lll}
\hline $\begin{array}{l}\text { Number of suspected reactions } \\
\text { by selected SOC [19] }\end{array}$ & ChAdOx1 nCoV-19 & Tozinameran \\
\hline General & 251,191 & 88,915 \\
Nervous system & 177,777 & 61,595 \\
Respiratory system & 28,291 & 15,341 \\
Cardiac & 9706 & 6428 \\
Blood & 7528 & 12,214 \\
All fatal reports & 1112 & 577 \\
Total reactions & 836,957 & 357,048 \\
Total reactions/million doses & 17,081 & 8152 \\
\hline
\end{tabular}

Reports can list several reactions, each of which is counted separately in the database

MHRA UK Medicines and Healthcare products Regulatory Agency, SOC system organ class between 3 and 73 reports per million doses for tozinameran (Fig. 2).

Table 1 shows the numbers of reported reactions for the two vaccines, and Table 2 presents the reactions by major system organ classes [19]. Overall, the number of fatal reports was 13 per million doses for tozinameran and 23 per million doses for ChAdOx1 nCoV-19.

There were 298 Yellow Card reports of CVST for ChAdOx1 nCoV-19 (Fig. 3a), 29 of which were fatal, and 59 similar reports, four fatal, for tozinameran by 27 October 2021 (Fig. 3b).

There were 104 Yellow Card reports of suspected reactions leading to RVT with ChAdOx $1 \mathrm{nCoV}-19$ (Fig. 3c) and 33 with tozinameran (Fig. 3d). None of the cases attributed to ChAdOx $1 \mathrm{nCoV}-19$ and just two of the tozinameran cases were reported before 10 March 2021. 

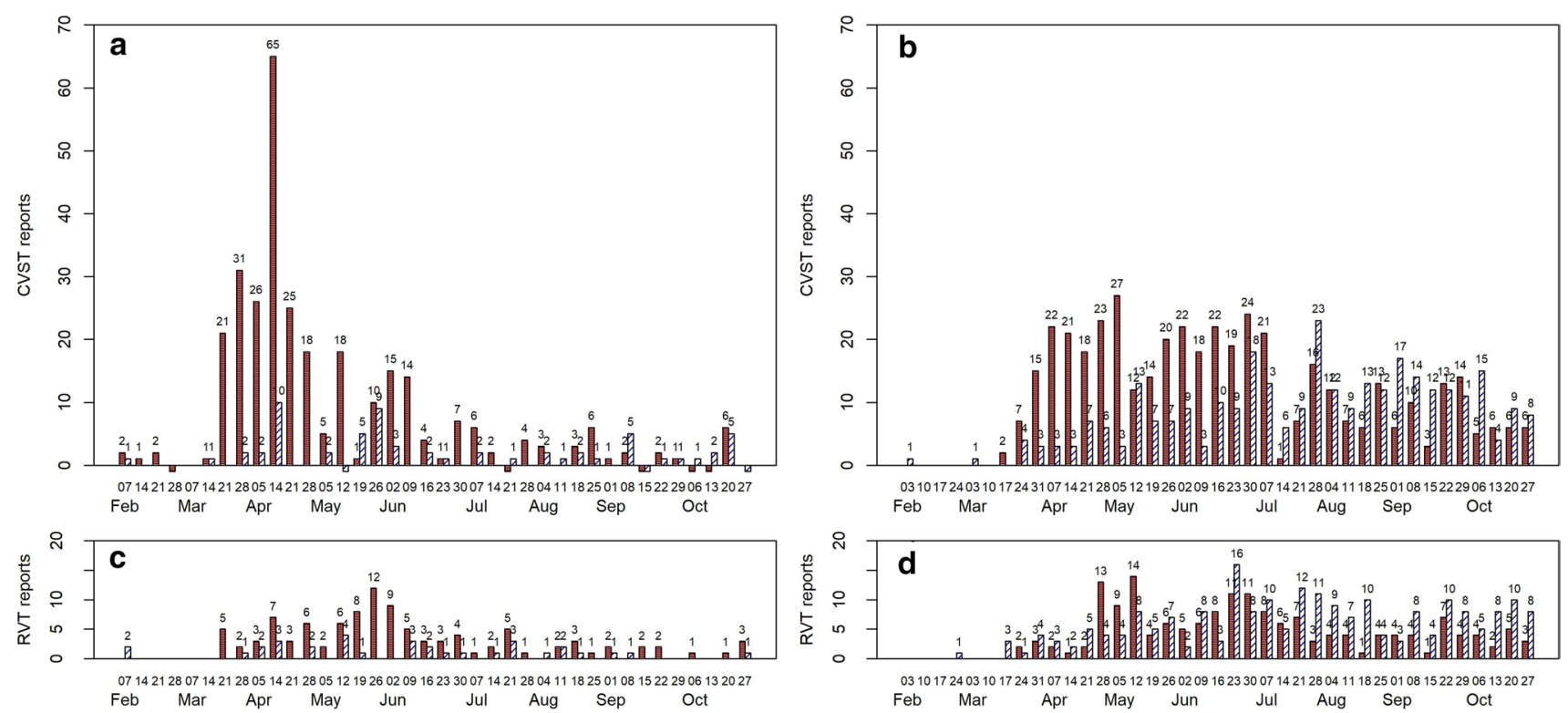

Fig. 3 a Incident numbers of Yellow Card reports of CVST for ChAdOx1 nCoV-19 and tozinameran at intervals; $\mathbf{b}$ total numbers of EudraVigilance European Economic Area reports of CVST at intervals; $\mathbf{c}$ total numbers of Yellow Card reports of RVT at intervals; $\mathbf{d}$ total numbers of EudraVigilance European Economic Area reports of RVT at intervals. The European Medicines Agency issued a press release on 10 March 2021 regarding three Austrian cases of thrombo-

\subsection{European Economic Area Data}

The EudraVigilance database contains fuller information on individual cases, since the age band, sex, and all the reactions reported for each case are available. Table 3 lists data abstracted from the spontaneous reports of suspected ADRs (relevant reports) that listed as reactions one or more of cerebral venous thrombosis, cerebral venous sinus thrombosis, superior sagittal sinus thrombosis, or transverse sinus thrombosis. The data are from 1 January to 31 October 2021. No reports of cerebral venous or sinus thrombosis were received for tozinameran in 2020.

EudraVigilance contained 443 EEA reports of CVST for ChAdOx1 nCoV-19, of which 72 were fatal, and 315 similar reports, 28 fatal, for tozinameran up to 31 October 2021. Of the 73 patients in the EEA reported to have had CVST who received ChAdOx $1 \mathrm{nCoV}-19$ and whose concomitant treatment was listed, three were taking aspirin, three enoxaparin, and two rivaroxaban, and one each took argatroban, apixaban, fondaparinux, phenprocoumon, and warfarin; 24 women were taking hormonal contraceptives or hormone replacement therapy.

Of the 66 patients in the EEA reported to have had CVST who received tozinameran and whose concomitant treatment was listed, one was taking aspirin with rivaroxaban, four others took aspirin, two warfarin, and one each acenocoumarol, clopidogrel, and dabigatran; 19 women were taking

sis after the ChAdOx1 nCoV-19 vaccine. Occasional points in the UK Medicines and Healthcare products Regulatory Agency Yellow Card data showed a decline in case numbers, perhaps because of recoding of suspected adverse drug reactions. CVST cerebral venous and sinus thrombosis, $R V T$ retinal vein occlusion and thrombosis, orange/red shading indicates ChAdOx1 $\mathrm{nCoV}-19$, blue/white shading indicates tozinameran

hormonal contraceptives or hormone replacement, and one vaginal levonorgestrel.

The EudraVigilance database distinguishes between reports from healthcare professionals and others. In total, $102(23 \%)$ of 443 relevant reports for ChAdOx $1 \mathrm{nCoV}-19$ and 94 (30\%) of 315 relevant reports for tozinameran were from non-healthcare professionals.

In the EEA by 31 October 2021, the total number of doses administered of ChAdOx $1 \mathrm{nCoV}-19$ was 68.8 million and of tozinameran 435.6 million. Approximately 5900 reports of suspected reactions per million doses had been logged for ChAdOx1 $\mathrm{nCoV}-19$ and 1300 reports per million doses for tozinameran.

The EudraVigilance database included 168 reports of RVT suspected to be associated with ChAdOx $1 \mathrm{nCoV}-19$, four with thrombocytopenia; 220 cases were suspected to be associated with tozinameran, none with thrombocytopenia.

\section{Discussion}

\subsection{Implications of the Data}

These publicly available data from two sources support the view that fatal suspected reactions to ChAdOx $1 \mathrm{nCoV}-19$ and tozinameran are very rare. Importantly, they avoid the need to estimate the relative reporting incidence indirectly, 
Table 3 Data from the EudraVigilance database for reports of suspected adverse drug reactions submitted from a country in the European Economic Area for the ChAdOx1 nCoV-19 and tozinameran vaccines from 1 January 2021 to 31 October 2021

\begin{tabular}{|c|c|c|}
\hline Vaccine & ChAdOx1 nCoV-19 & Tozinameran \\
\hline Total number of reports ${ }^{\mathrm{a}}$ & 406,132 & 549,762 \\
\hline Relevant reports & 443 & 315 \\
\hline Female sex & 291 & 196 \\
\hline \multicolumn{3}{|l|}{ Age, years } \\
\hline $0-17$ & 1 & 5 \\
\hline $18-64$ & 233 & 132 \\
\hline $65-85$ & 46 & 47 \\
\hline$>85$ & 3 & 9 \\
\hline Not specified & 8 & 3 \\
\hline Male & 150 & 117 \\
\hline \multicolumn{3}{|l|}{ Age, years } \\
\hline $12-17$ & 0 & 1 \\
\hline $18-64$ & 111 & 79 \\
\hline $65-85$ & 37 & 28 \\
\hline$>85$ & 0 & 6 \\
\hline Not specified & 2 & 3 \\
\hline Sex not specified & 2 & 2 \\
\hline \multicolumn{3}{|l|}{ Reports of fatal reactions } \\
\hline Female & 43 & 9 \\
\hline Male & 29 & 19 \\
\hline Total & 72 & 28 \\
\hline \multicolumn{3}{|c|}{ Thrombocytopenia or reduced platelet count or both also reported } \\
\hline Female & 120 & 2 \\
\hline Male & 63 & 7 \\
\hline Total & 183 & 9 \\
\hline
\end{tabular}

Relevant reports cite reactions as one or more of cerebral venous sinus thrombosis, cerebral venous thrombosis, superior sagittal sinus thrombosis, and transverse sinus thrombosis

${ }^{a}$ Up to 6 November 2021

because of the availability of data on the number of doses administered. They demonstrate the association between adverse publicity and the reporting rate for ADRs. They also provide a signal of a possible link between both vaccines and rare and unusual thrombotic events in the intracranial venous circulation and retinal vein thrombosis, in the absence of thrombocytopenia.

ADRs that occur in fewer than 1 in 1000 people are considered rare, and most medicines are marketed on the basis of clinical trials that are too small to detect rare or very rare risks of harm. The detection of very rare adverse reactions relies initially on detection of pharmacovigilance 'signals' [20], often by examination of databases of spontaneously reported suspicions of ADRs, such as the MHRA's Yellow Card database [21] and the EMA's EudraVigilance database [22]. Furthermore, clinical trials exclude groups of patients who may be especially susceptible to ADRs, such as pregnant or elderly people and those with co-morbid conditions.

The rates of spontaneous reporting of adverse reactions to ChAdOx1 nCoV-19 and tozinameran have fluctuated since the introduction of the vaccines into clinical use in the UK. Especially high rates were observed for total reports on ChAdOx1 nCoV-19 in February and for total and fatal reports on both $\mathrm{ChAdOx} 1 \mathrm{nCoV}-19$ and tozinameran in March 2021. One possible explanation for these peaks in reporting is negative publicity in the press. For example, one UK newspaper carried the headline "Five countries halt use of one batch of AstraZeneca jab after fatal blood clot" on 10 March 2021 [23]. Reporting in the Yellow Cards database of CVST for ChAdOx1 nCoV-19 increased rapidly after that date (Fig. 3). The proportion of people being given a second dose of tozinameran had started to increase above $20 \%$ in the last week of March 2021, with little evidence of a change in total reporting rate; the proportion of vaccinees given a second dose of ChAdOx $1 \mathrm{nCoV}-19$ reached $20 \%$ only in the last weeks of April, again with little evidence of an effect on reporting of suspected ADRs. This makes an alternative explanation-that adverse reactions were more common after the second dose of a vaccine-unlikely.

Data from the EEA for the period 1 January 2021 to 31 July 2021 showed total reports for ChAdOx1 nCoV-19 at similar rates to those in the UK but lower rates of reports for tozinameran. Reports of CVST as a suspected adverse reaction were more common for ChAdOx $1 \mathrm{nCoV}-19$. Of 443 reports, 183 (41\%) were associated with thrombocytopenia. There were 315 reports of CVST to tozinameran, but only nine $(2.9 \%)$ of these were associated with thrombocytopenia. There were also several EudraVigilance reports of retinal vein thrombosis with both vaccines, but very few cases were associated with thrombocytopenia. This raises the possibility that the vaccines can cause two different forms of cerebral venous thrombotic events and that, while onewhich is primarily associated with ChAdOx $1 \mathrm{nCoV}-19$ - is accompanied by thrombocytopenia, the other-which can occur with both vaccines - is not. An alternative explanation is that, especially after 10 March 2021, reports of suspected associations represented adverse events that were not ADRs. One important confounder is COVID-19, since the illness itself is associated with thrombotic events.

\subsection{Limitations}

The data we used in our study came from spontaneous reports of suspected ADRs. As the MHRA states, "A report of a suspected ADR to the Yellow Card scheme does not necessarily mean that it was caused by the vaccine, only that the reporter has a suspicion it may have. Underlying or previously undiagnosed illness unrelated to vaccination can also be factors in such reports. The 
relative number and nature of reports should therefore not be used to compare the safety of the different vaccines" $[12,17]$. Spontaneous reports are subject to confounding and biases, the most important of which include underreporting of suspected adverse reactions and reporting of events that are associated with treatment but not caused by it. Novel ADRs can easily be missed in practice and may be reported to regulators only after they have been publicized in the medical literature [24]. By contrast, wide publicity can stimulate reporting $[25,26]$. Thus, the data we reviewed constitute signals of possible ADRs, which would require formal studies - which might or might not be confirmatory. An example of the former is practolol, which was shown to be associated with an oculomucocutaneous syndrome, reports of which increased after an initial observation [27]. An example of the latter is montelukast, which was suspected of being associated with an increased risk of suicide following an initial report [28], an association that has not subsequently been confirmed [29]. In our analysis of the reports of suspected ADRs to the SARS-CoV-2 vaccines, the rapid accumulation of reports of CVST after the initial publicity strongly supports the view that novel ADRs can initially be missed and that adverse publicity leads to a rapid increase in reporting.

A few reports about vaccines against SARS-CoV-2 did not specify which of the vaccines was given. The syndrome of VITT may occur more often in younger people; in the vaccination campaign in the UK, patients were recruited by age, so susceptibility may have differed in cohorts recruited at different times. Some reports in the EudraVigilance database, and an unknown number of reports in the Yellow Card database, were submitted by people who were not healthcare professionals. While such reports can be helpful [30, 31], they may not be comparable to reports from clinically trained personnel. We used weekly reports to provide both the numerator (number of reactions) and the denominator (number of vaccine doses administered), but we cannot be sure that the date of report registration corresponds with the date of the suspected reaction or of the delay between immunization and reaction. In consequence, variation in the denominator may account for some of the variation in the weekly reporting rate.

\section{Conclusion}

Reports of fatal adverse reactions to coronavirus vaccines are very rare. Cerebral venous and sinus thrombosis has been associated with both $\mathrm{ChAdOx} 1 \mathrm{nCoV}-19$ and tozinameran, although the spontaneous reporting rate with the latter is about one-quarter of that with the former. Reporting increased after the reaction was first suggested. Very few of the cases associated with tozinameran were accompanied by thrombocytopenia. This raises the possibility of two mechanisms for thrombotic reactions to these vaccines. Rates of any form of CVST are so low that they would not be reliably detected by any form of standard clinical trial.

Acknowledgements The authors thank Professor Philip Murray of the University of Birmingham for his most helpful advice on RVT.

\section{Declarations}

Funding No sources of funding were used to assist in the preparation of this article.

Conflict of interest All authors have completed the ICMJE uniform disclosure form. JKA and REF have both written widely about adverse drug reactions and interactions and have from time to time received fees for legal reports, payments for articles, and royalties on books that they have written on the subject. The West Midlands Centre for Adverse Drug Reactions is associated with the Yellow Card Centre West Midlands, which receives funds from the MHRA. CA has no other conflicts of interest relevant to this article. RS is a member of the Central Advisory Committee, and an Electronic Review Committee, which advise the MHRA on governance of the Clinical Practice Research Datalink; the views expressed here do not reflect that role or that of the MHRA. All authors assert that the views expressed in this article are their own and do not necessarily reflect those of any organization with which they may be associated.

Ethics approval Not applicable.

Consent to participate Not applicable.

Consent for publication Not applicable.

Availability of data and material Publicly available data were used for this research.

Code availability Not applicable.

Author contributions All authors contributed to the design of the study and the analysis of the results. CA and REF retrieved the data for the study. REF wrote the first draft of the manuscript. RS constructed the graphs. All authors contributed to manuscript revision and read and approved the submitted version.

\section{References}

1. Polack FP, Thomas SJ, Kitchin N, et al. Safety and efficacy of the BNT162b2 mRNA Covid-19 vaccine. N Engl J Med. 2020;383(27):2603-15.

2. Voysey M, Clemens SAC, Madhi SA, et al. Safety and efficacy of the ChAdOx1 nCoV-19 vaccine (AZD1222) against SARS-CoV-2: an interim analysis of four randomised controlled trials in Brazil, South Africa, and the UK. Lancet. 2021;397(10269):99-111.

3. Newcombe RG, Altman DG. Chapter 6. Proportions and their differences. In: Altman DG, Machin D, Bryant TN, Gardner MJ, editors. Statistics with confidence. 2nd ed. London: BMJ Books; 2000. 
4. Council for International Organizations of Medical Sciences. Guidelines for preparing core clinical-safety information on drugs. 2nd ed. Geneva: CIOMS; 1999.

5. Weber JCP. Mathematical models in adverse drug reaction assessment. In: D'Arcy PF, Griffin JP, editors. Iatrogenic diseases. 3rd ed. Oxford: Oxford University Press; 1986.

6. European Medicines Agency. COVID-19 Vaccine AstraZeneca: PRAC preliminary view suggests no specific issue with batch used in Austria. London: EMA. https://www.ema.europa.eu/en/news/ covid-19-vaccine-astrazeneca-prac-preliminary-view-suggests-nospecific-issue-batch-used-austria. Accessed 5 June 2021.

7. BBC News. Oxford-AstraZeneca: EU says 'no indication' vaccine linked to clots. https://www.bbc.co.uk/news/world-europe-56357 760. Accessed 5 June 2021.

8. British Society for Haematology. Guidance produced by the Expert Haematology Panel Focussed on Vaccine Induced Thrombosis and Thrombocytopenia (VITT). https://b-s-h.org.uk/aboutus/news/guidance-produced-by-the-expert-haematology-panelehp-focussed-on-vaccine-induced-thrombosis-and-thrombocyt openia-vitt/. Accessed 3 Nov 2021.

9. Scully M, Singh D, Lown R, et al. Pathologic antibodies to platelet factor 4 after ChAdOx1 $\mathrm{nCoV}-19$ vaccination. N Engl J Med. 2021;384(23):2202-11. https://doi.org/10.1056/NEJMoa2105385.

10. Greinacher A, Thiele T, Warkentin TE, Weisser K, Kyrle PA, Eichinger S. Thrombotic thrombocytopenia after ChAdOx1 nCov19 vaccination. N Engl J Med. 2021;384(22):2092-101.

11. Bauman MMJ, Naylor RM, Wijdicks EF. HIT in the head: a systematic review of cerebral venous sinus thrombosis in classical and autoimmune heparin-induced thrombocytopenia. J Thromb Thrombolysis. 2021;52(3):952-61. https://doi.org/10.1007/ s11239-021-02484-6.

12. Medicines \& Healthcare products Regulatory Agency. Research and analysis. Coronavirus vaccine-weekly summary of Yellow Card reporting. https://www.gov.uk/government/publications/ coronavirus-covid-19-vaccine-adverse-reactions/coronavirusvaccine-summary-of-yellow-card-reporting. Accessed 3 Nov 2021.

13. Nguyen QD, Van Do D, Feke GT, Demirjian ZN, Lashkari K. Heparin-induced antiheparin-platelet antibody associated with retinal venous thrombosis. Ophthalmology. 2003;110(3):600-3.

14. European Centre for Disease Prevention and Control. COVID19 Vaccine rollout review. https://covid19-vaccine-report.ecdc. europa.eu/. Accessed 6 Nov 2021.

15. European Union. Size and population. 2021. https://europa.eu/ european-union/about-eu/figures/living_en\#size-and-population. Accessed 6 Nov 2021

16. Worldometer. Countries in the world by population. 2021. https:// www.worldometers.info/world-population/population-by-count ry/. Accessed 3 Nov 2021.

17. Medicines \& Healthcare products Regulatory Agency. COVID19 AstraZeneca Vaccine Analysis Print. https://assets.publishing. service.gov.uk/government/uploads/system/uploads/attachment_ data/file/989997/COVID-19_vaccine_AstraZeneca_analysis_ print.pdf. Accessed 5 June 2021.

18. Medicines \& Healthcare products Regulatory Agency. COVID-19 mRNA Pfizer-BioNTech Vaccine Analysis Print. https://assets. publishing.service.gov.uk/government/uploads/system/uploads/ attachment_data/file/989996/COVID-19_mRNA_Pfizer-_BioNT ech_Vaccine_Analysis_Print.pdf. Accessed 3 Nov 2021.

19. The Medical Dictionary for Regulatory Activities (MedDRA). https://www.meddra.org/about-meddra/organisation. Accessed 12 June 2021.

20. Hauben M, Aronson JK. Defining 'signal' and its subtypes in pharmacovigilance based on a systematic review of previous definitions. Drug Saf. 2009;32(2):99-110.

21. YellowCard Interactive Drug Analysis Profiles. https://yellowcard. mhra.gov.uk/iDAP/. Accessed 3 Nov 2021.

22. Eudravigilance - European database of suspected adverse drug reaction reports. https://www.adrreports.eu/en/. Accessed 6 Nov 2021.

23. de La Mare T. Five countries halt use of one batch of AstraZeneca jab after fatal blood clot. Evening Standard 2021 Mar 10. https:// www.standard.co.uk/news/uk/astrazeneca-austria-latvia-luxem bourg-lithuania-b923420.html. Accessed 18 Jan 2022.

24. Wright P. Untoward effects associated with practolol administration: oculomucocutaneous syndrome. $\mathrm{Br}$ Med $\mathrm{J}$. 1975;1(5958):595-8.

25. Martin RM, May M, Gunnell D. Did intense adverse media publicity impact on prescribing of paroxetine and the notification of suspected adverse drug reactions? Analysis of routine databases, 2001-2004. Br J Clin Pharmacol. 2006;61:224-8.

26. Postma LGM, Donyai P. The cooccurrence of heightened media attention and adverse drug reaction reports for hormonal contraception in the United Kingdom between 2014 and 2017. Br J Clin Pharmacol. 2021;87(4):1768-77.

27. Wright P. Skin reactions to practolol. Br Med J. 1974;2(5918):560.

28. Iessa N, Star K, Wilton L, Curran S, Edwards IR, Aronson JK, Murray M, Besag FM, Wong IC. Montelukast and suicide: causality assessment using spontaneous reports and Bradford Hill guidelines. Drug Saf. 2011;34(10):949-50.

29. Sansing-Foster V, Haug N, Mosholder A, et al. Risk of psychiatric adverse events among montelukast users. J Allergy Clin Immunol Pract. 2021;9(1):385-393.e12.

30. de Langen J, van Hunsel F, Passier A, et al. Adverse drug reaction reporting by patients in the Netherlands: three years of experience. Drug Saf. 2008;31:515-24.

31. Avery AJ, Anderson C, Bond CM, et al. Evaluation of patient reporting of adverse drug reactions to the UK "Yellow Card Scheme": literature review, descriptive and qualitative analyses, and questionnaire surveys. Health Technol Assess. 2011;15(20):1234, iii-iv. 